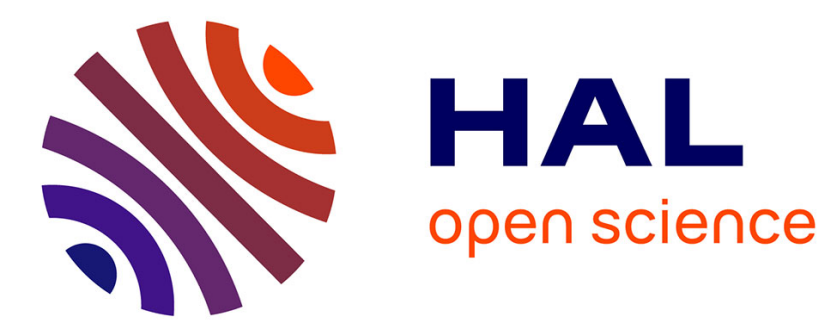

\title{
Coupling of rotating water jets by surface waves
}

\author{
F. Giorgiutti, L. Laurent, F. Daviaud
}

\section{To cite this version:}

F. Giorgiutti, L. Laurent, F. Daviaud. Coupling of rotating water jets by surface waves. Physical Review E: Statistical, Nonlinear, and Soft Matter Physics, 1998, 58, pp.512-521. 10.1103/PhysRevE.58.512 . cea-01373943

\section{HAL Id: cea-01373943 https://hal-cea.archives-ouvertes.fr/cea-01373943}

Submitted on 29 Sep 2016

HAL is a multi-disciplinary open access archive for the deposit and dissemination of scientific research documents, whether they are published or not. The documents may come from teaching and research institutions in France or abroad, or from public or private research centers.
L'archive ouverte pluridisciplinaire HAL, est destinée au dépôt et à la diffusion de documents scientifiques de niveau recherche, publiés ou non, émanant des établissements d'enseignement et de recherche français ou étrangers, des laboratoires publics ou privés. 


\title{
Coupling of rotating water jets by surface waves
}

\author{
F. Giorgiutti,* L. Laurent, and F. Daviaud \\ Commissariat à l'Energie Atomique (CEA), Service de Physique de l'Etat Condensé, Saclay, F-91191 Gif sur Yvette Cedex, France
}

(Received 8 July 1997; revised manuscript received 23 February 1998)

\begin{abstract}
Above a critical flow rate, a single jet impinging from below on a water-air interface oscillates while arrays of jets exhibit collective behaviors. The aim of the paper is to explain the physics governing such an array. First, a series of experiments shows that the instability mechanism leading to the oscillation of a single jet is due to a feedback effect between surface distortion and the unstable jet as in the "jet-edge" system and that several jets are coupled through surface waves. Then a modelization is proposed in which jets are considered as oscillators close to their limit cycle coupled by delayed action to take into account the wave propagation. A good agreement is obtained between the predictions of the model and experiments on sets of two or four jets. The long range of the surface waves and the large number of oscillators render this jet configuration very similar to idealized phase coupled nonlinear oscillators investigated in theoretical models but with a not instantaneous coupling ensured by traveling waves. This allows in particular the existence of various synchronization modes, which appear as geometrical patterns. [S1063-651X(98)07407-8]
\end{abstract}

PACS number(s): 47.20. $-\mathrm{k}, 68.15 .+\mathrm{e}, 68.10 .-\mathrm{m}$

\section{INTRODUCTION}

A lot of theoretical work has been done on large populations of coupled oscillators because of their interest to represent various physical or biological systems with a large number of degrees of freedom (see, for instance, [1-3]). On the experimental side, several attempts have been made to design extended systems that can be considered as such a set of oscillators. Some of them use fluids perturbed by a spatially periodic action that to some extent behave as coupled oscillators such as coupled wakes [4], air jets [5], magnetically driven vortices [6], and to a lesser extent coupled thermal boundary layers [7]. Modelization of these systems has often been done by using a nonlinear amplitude equation modified to take into account the coupling with the neighboring oscillators. The exact nature of the coupling term depends on the underlying physics and various schemes have been used such as an additional drive depending linearly on neighboring amplitude $[4,6]$, an additional nonlinear term [6], or a delayed modification of the nonlinear saturation term $[5,7]$. Another interesting system consists in using vertical jets close to a water-air interface. It can be easily extended to configurations with a large number of oscillators. When they are isolated, such jets can rotate or oscillate at constant amplitude [8,9]. When they are associated in arrays, phase locks and a wide variety of phase patterns are observed that depend on the symmetries of the network [10].

Up to now, the understanding of the jet rotary instability was only partial, in particular the observed frequency dependence (decreasing with the jet velocity), which is quite unusual for standard jet instability. Moreover, no quantitative model for the coupling and the phase pattern has been proposed. The two aims of this paper are (i) to provide a clearer understanding of the jet rotation and (ii) to present the first model able to predict quantitatively the coupling between

\footnotetext{
*Present address: Laboratoire FAST, Bâtiment 502, Campus Universitaire, 91405 Orsay Cedex, France.
}

jets. In the next section, after a brief presentation of the experimental setup, experiments concerning the single jet instability $[11,12]$ are presented. A model of the oscillation is discussed based on the "jet tone" concept [13], which provides the right scaling of the frequency. Then, in Sec. III, simple arrays of two or four jets are investigated experimentally and a model is presented to explain the observed phase patterns. In Sec. IV, the results are discussed. The model is tested by comparing the predicted and observed frequency and modes of oscillation for various sizes and flow rates. Then to explain the unlocking of jets when they are separated by a too large distance, the effect of a slight detuning of the jets is investigated.

\section{PROPERTIES OF THE JET AS AN OSCILLATOR}

\section{A. Experimental setup}

Figure 1 describes the experimental setup. The jets flow out of a tranquilization chamber containing a 5-cm-thick porous medium. The chamber is filled by pressurized water

photodiode video camera
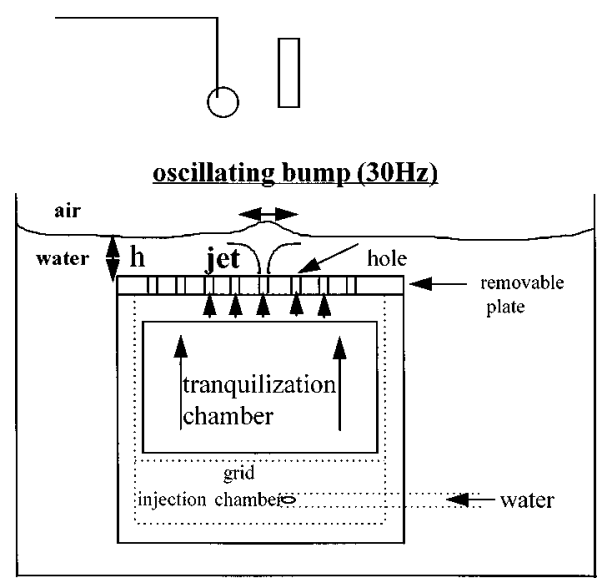

FIG. 1. The experimental setup. 


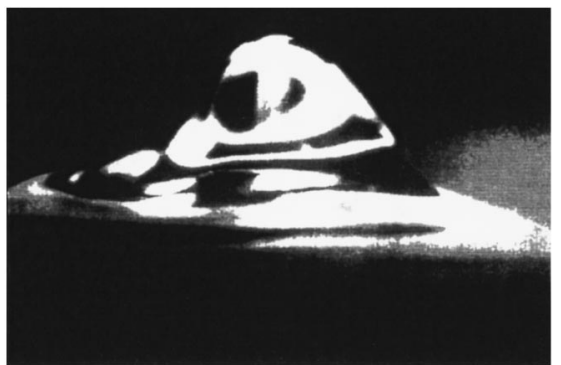

FIG. 2. Picture of a rotating bump for $U>U_{c}$. The jet diameter is equal to $1 \mathrm{~mm}$, the height and width of the bump are of the same order, about $3 \mathrm{~mm}$.

supplied from an elevated reservoir (to avoid any vibration due to a pump) through a flow meter and a regulation valve. Its top part is a removable 6-mm-thick perforated plate located a few millimeters below the surface. Various types of plates can be used. For the present paper, square networks of holes with a radius $b=0.5 \mathrm{~mm}$ are used and flow velocities at the hole, and $U$ lies between 0.5 and $0.7 \mathrm{~m} \mathrm{~s}^{-1}$ leading to the Reynolds numbers $(U b / \nu)$ in the range 250-350. The whole device is immersed in a 1101 plexiglass constant level water tank. In some experiments the surface tension was varied in the range $50-75 \times 10^{-3} \mathrm{Nm}^{-1}$ using soap. To control the depth $h$ of the jets below the surface (typically $6 \mathrm{~mm}$ ), the vertical position of the jet nozzles can be adjusted with a micropositioner. More details can be found in [10]. When one of the jets reaches the water-air interface, there is a stationary axisymmetric bump. Its radius $b^{\prime}$ is determined by the equilibrium between the jet pressure $\rho U^{\prime 2}$ (where $\rho$ is the water volumic mass and $U^{\prime}$ is the jet velocity at the bump) and the surface resistance, which scales as $\sigma / b^{\prime}$ ( $\sigma$ is the superficial tension). When $U$ is larger than a threshold $U_{f}$ so that this equilibrium is no longer possible, i.e., $U^{\prime}$ is so large that equilibrium would require $b^{\prime}$ smaller than $b$, the surface breaks and the system looks like a "small fountain."

\section{B. Observations}

Above a critical velocity $U=U_{c}$ the axisymmetric equilibrium of the bump becomes unstable and it starts rotating around the jet axis (Fig. 2), while generating surface waves with a spiral geometry (Fig. 3). The frequency range of these oscillations lies between 10 and $50 \mathrm{~Hz}$, and measurements can be made either with stroboscopic lighting for visualization or intensity of a light beam reflecting on the bump towards a photodiode for time evolution. Experimental results

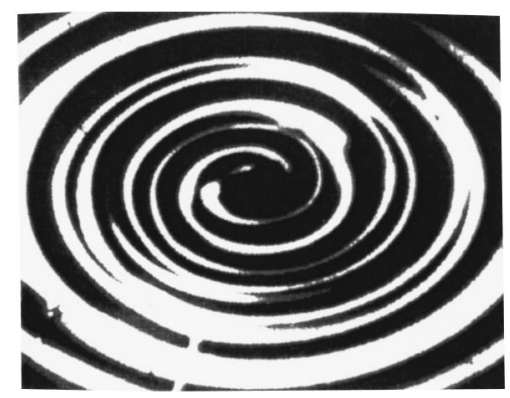

FIG. 3. Picture from above of the surface waves generated by the rotating bump (spiral waves). about frequency can be summarized in the following way: (i) for a given geometry the frequency decreases with the jet velocity [Fig. 4(a)], (ii) it increases with surface tension [Fig. 4(b)], (iii) when the depth, or $U$, is varied, the most relevant parameter seems to be the bump height $b^{\prime}$ (where primes denotes quantities related to the bump) as shown in Fig. 4(b) of [10].

It appears that the jet rotation can be synchronized when excited with surface waves in the range of frequencies corresponding to the free rotation one. This was shown in a series of experiments, which present some similarities with the oscillating edge experiment by Staubli and Rockwell [13]. Surface waves were generated on the water tank through a blade connected to the coil of a loudspeaker. The evolution of the bump oscillation with the forcing frequency has been followed. Two phase-locking areas have been seen for forcing frequencies close to the bump frequency or in a region at lower frequencies. For other frequencies both oscillations, i.e., forcing waves and bump oscillation coexist (see Fig. 5).

\section{Discussion}

The observed rotation of the bump suggests that the jet is subjected to a helical instability. This is compatible with the Reynolds number range. Moreover, previous works demonstrated that a few diameters away from the nozzle, when the shear layer has widened, this mode is the one with the largest growth rate $[11,12]$. However, for the free jet, the most unstable $\omega$ are of order of $0.5 \mathrm{U} / \mathrm{b}$, which corresponds to frequencies of the order of $100 \mathrm{~Hz}$ for the present experiment, i.e., typically three times the experimental ones. In addition, the observed frequency decreases with jet velocity instead of increasing. An additional frequency-selection mechanism must be invoqued. The water-air interface, which is not in the standard theory, provides this ingredient. The helical instability distorts the surface and this causes a pressure fluctuation along the jet path, which can be amplified by the jet. Such an amplification is well known and examples are given in $[14,15]$. Indeed the strength of the feedback will depend on the amplitude of the surface distortion. When the helical instability distorts the bump, surface tension tends to restore the initial shape, which corresponds to the equilibrium between the surface tension and the pressure. The spring force scales as $\sigma b^{\prime}$, i.e., the product of the surface tension by a characteristic length. To restore the initial shape, this force has to modify the momentum of the water inside the bump, which scales as $\rho U^{\prime} b^{\prime 3}$. The characteristic time of the bump relaxation is the ratio of these two quantities, i.e., $\tau$ $=\rho U^{\prime} b^{\prime 2} / \sigma$. Thus the feedback between the bump and the jet is expected to be optimum when the latter rotates at an angular velocity $\omega_{0}$, so that $\omega_{0} \tau_{r}=1$, i.e., $\omega_{0}$ is proportional to $\sigma / \rho b^{\prime 2} U^{\prime}$.

Using the flow rate conservation $b^{\prime 2} U^{\prime}=b^{2} U$, the scaling of frequency should be $\omega_{0}=G(U) \sigma / \rho b^{2} U$, where $G(U)$ is an unknown dimensionless number that takes into account the numerical factor and the actual geometry of the flow, which may vary with the jet velocity. In Fig. $6, \rho b^{2} U \omega_{0} / \sigma$ [i.e., the expected $G(U)]$ is plotted as a function of the velocity of the jet for various water velocities and depth. The fact that this quantity is almost constant (except for high $U$, 

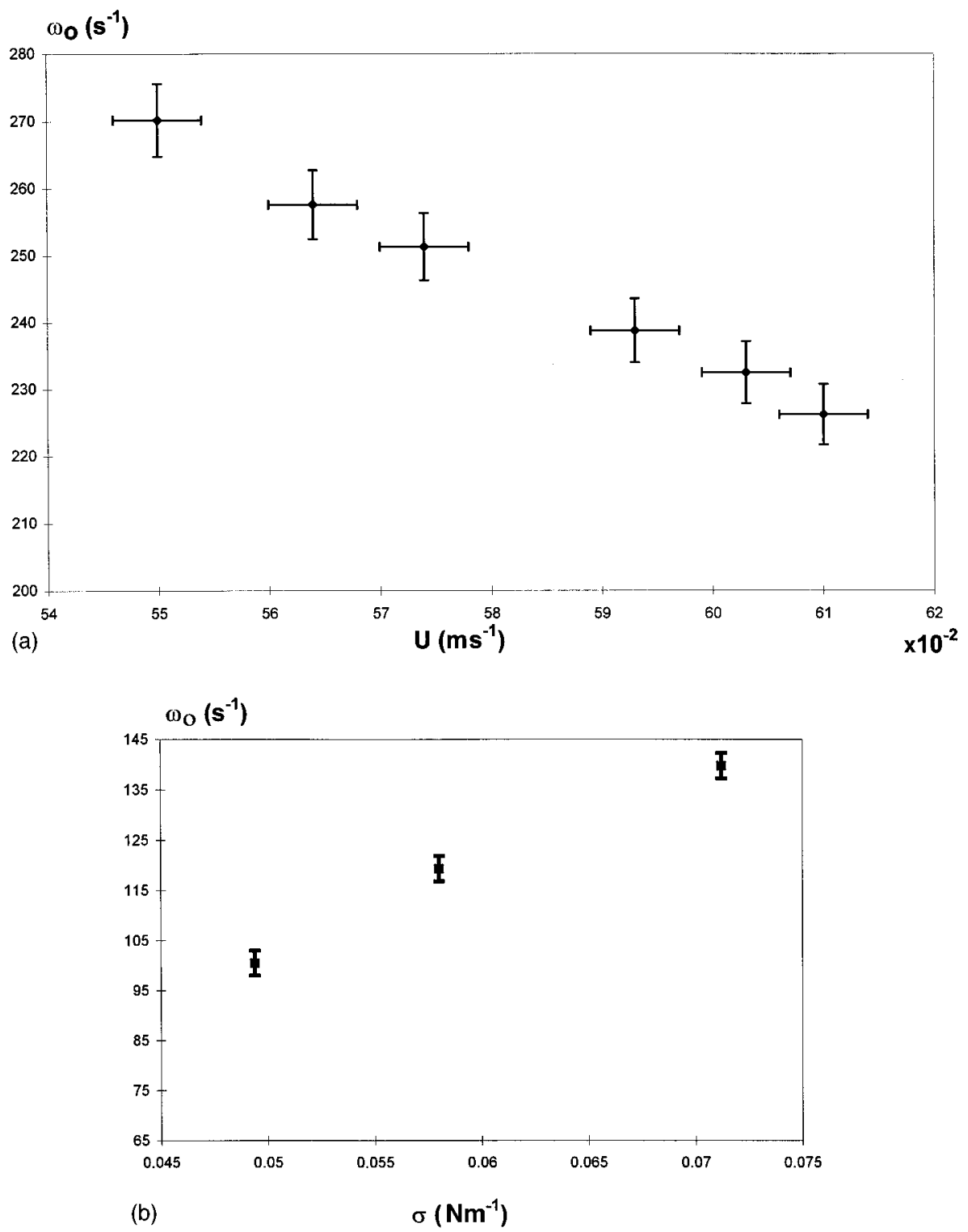

FIG. 4. (a) Evolution of $\omega_{0}$ with the jet velocity $U$. (b) Effect on $\omega_{0}$ of a change in surface tension obtained by adding soap to water.

i.e., low $\omega_{0}$ ) shows that the model for oscillation is correct and moreover that geometry distortion due to variations of $U$ is not too large. In conclusion, taking into account the value of $G$ deduced from Fig. 6 one gets

$$
\omega_{0}=(0.9 \pm 0.1) \sigma / \rho b^{2} U .
$$

It is also possible to eliminate $U^{\prime}$ in the relation $\omega_{0}$ $\approx \sigma / \rho b^{\prime 2} U^{\prime}$ using the equilibrium discussed in Sec. II A between the stress $\rho U^{\prime 2}$ and superficial tension $\sigma / b$, i.e., $U^{\prime}$ is proportional to $b^{-0.5}$ so that $\omega_{0}$ should approximatively scales with $b^{-1.5}$. This curve is compatible with experimental results [10] although the exponent cannot be determined accurately (between -2 and -1.5 ).

As discussed at the beginning of the section, these frequencies are two or three times lower than the optimum for the amplification by the jet. However, taking a reasonable velocity profile across the jet, the linear theory still predicts an instability with $\operatorname{Re}\left(k_{z}\right)$ and $\operatorname{Im}\left(k_{z}\right)$ of the order of 1000 and
$100 \mathrm{~m}^{-1}$, respectively [12]. The amplification takes place on distances $l$ lying between $b^{\prime}$ and $h$ so that its value $e^{\operatorname{Im}\left(k_{z}\right) 1}$ will be in the range $1-1.5$. If the coupling between the pressure modification due to the bump distortion and the jet is not too lossy, the system can oscillate. To explain the rotation start at $U=U_{c}$, the following scenario is likely: when $U$ is low, the Reynolds number is too low for an amplification process to take place. When $U$ increases above $U_{c}, \operatorname{Im}\left(k_{z}\right)$ reaches a value such that the oscillation is possible. The most favored frequency is $\omega_{0}$ since the feedback on pressure is optimum at the bump resonance.

The loudspeaker experiment can be interpreted along the same lines. When the incoming waves distorts the bump efficiently i.e., when its frequency is close to $\omega_{0}$ it can lock in phase the jet instability. This is not contradictory with the presence of another region at lower frequency, where there is a phase locking that could correspond to the direct excitation of the jet by the surface wave. 


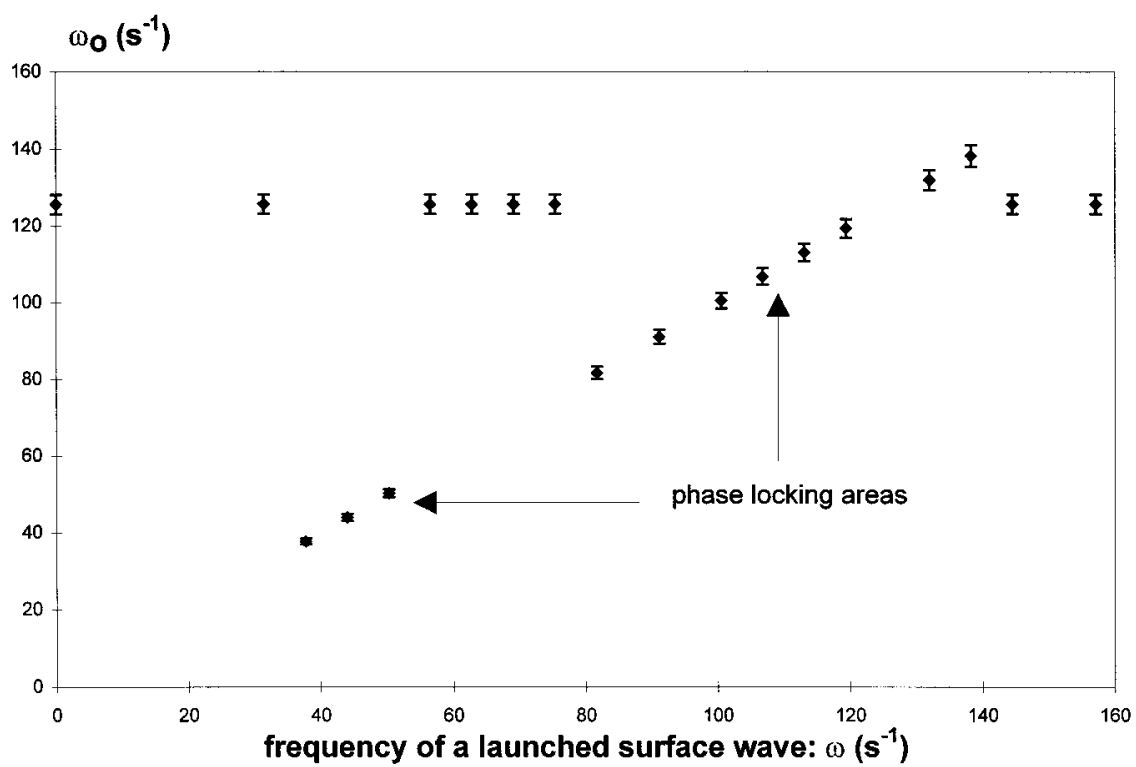

FIG. 5. Effect on the bump pulsation $\omega_{0}$ of a launched surface wave (blade connected to a loudspeaker excited by a sinusoidal tension). The natural value of $\omega_{0}$ is $126 \mathrm{~s}^{-1}$.

\section{COUPLING OF OSCILLATORS IN SIMPLE GEOMETRIES}

\section{A. Experimental results with simple arrays}

Results of Sec. II suggest that when the system contains several jets, they can be coupled. This has been investigated for two types of jet configurations.

(i) The first one consists in two jets separated by distances $6 \times 10^{-3}<a<12 \times 10^{-3} \mathrm{~m}$, the plate being $6 \mathrm{~mm}$ deep. When $a$ is too large (above $12 \times 10^{-3} \mathrm{~m}$ ) the coupling disappears. The flow rate was varied, limited on the lower side by the threshold for jet oscillation, and on the upper side by the fountain regime. This corresponds to the single jet frequency range: $240<\omega_{0}<300 \mathrm{~s}^{-1}$ [Eq. (1)]. The two bumps oscillate at the same frequency, with the same amplitude (note that the photodiode technique does not allow an accu- rate amplitude measurement) and the same direction of rotation. There are two differences with the single jet case: (i) the critical flow rate for the bump oscillation appearance is lower for two bumps than the one for a single one, (ii) the frequency $\omega$ is different from the single jet one $\omega_{0}$ as shown in Fig. 7. The results obtained during $\omega_{0}$ scans (i.e., $U$ scans since $\omega_{0}$ decreases as $U$ increases) for various values of jet spacing $a$, are presented in Table I. The second column represents the phase difference between the motion of the two jets. Two collective modes are observed: an acoustic mode where the two bumps oscillate in phase (phase difference of $2 \pi$ ), and an optical mode where the two bumps oscillate in phase opposition (phase difference of $\pi$ ). When there is a transition, the symbol at left (right) represents the mode at low (high) $\omega_{0}$. In this case the value of $\omega_{0}$ at the transition is displayed in the third column. A transition between two

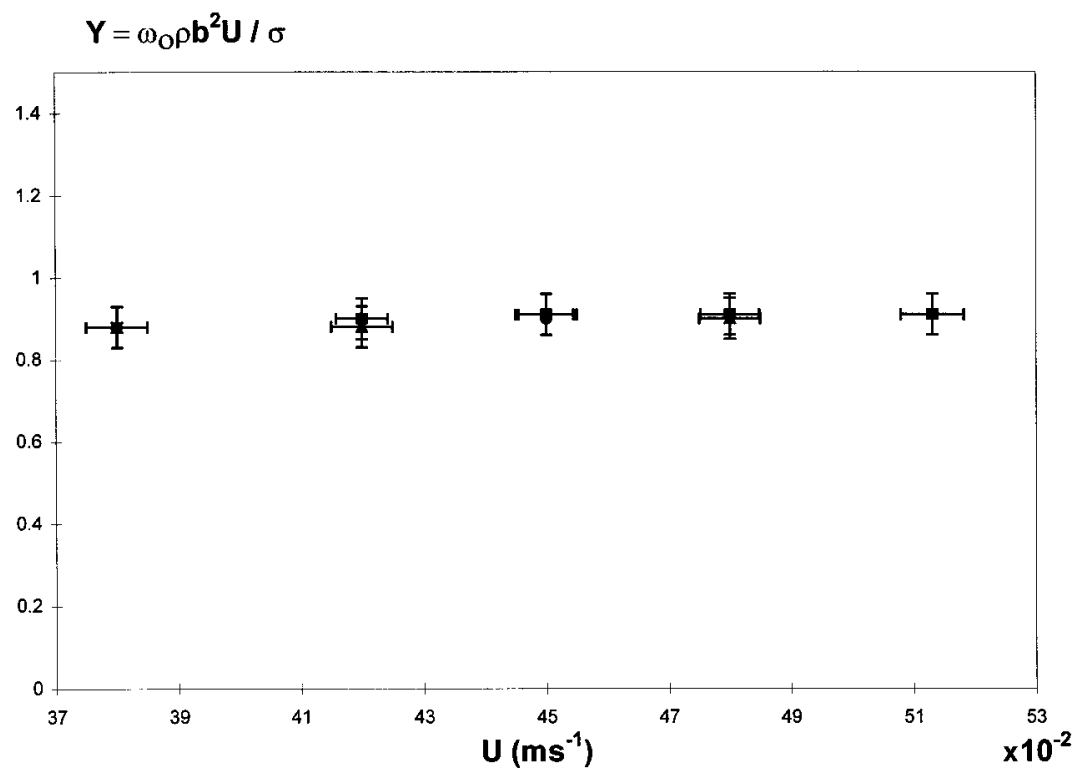

FIG. 6. Evolution of the rescaled pulsation $Y=\rho \omega_{0} b^{2} U / \sigma$ with the jet velocity $U$ for one single jet, for different depths. The figure contains values obtained for plate depths $=6,7$, and $10 \mathrm{~mm}$. 
$\omega\left(\mathbf{s}^{-1}\right)$

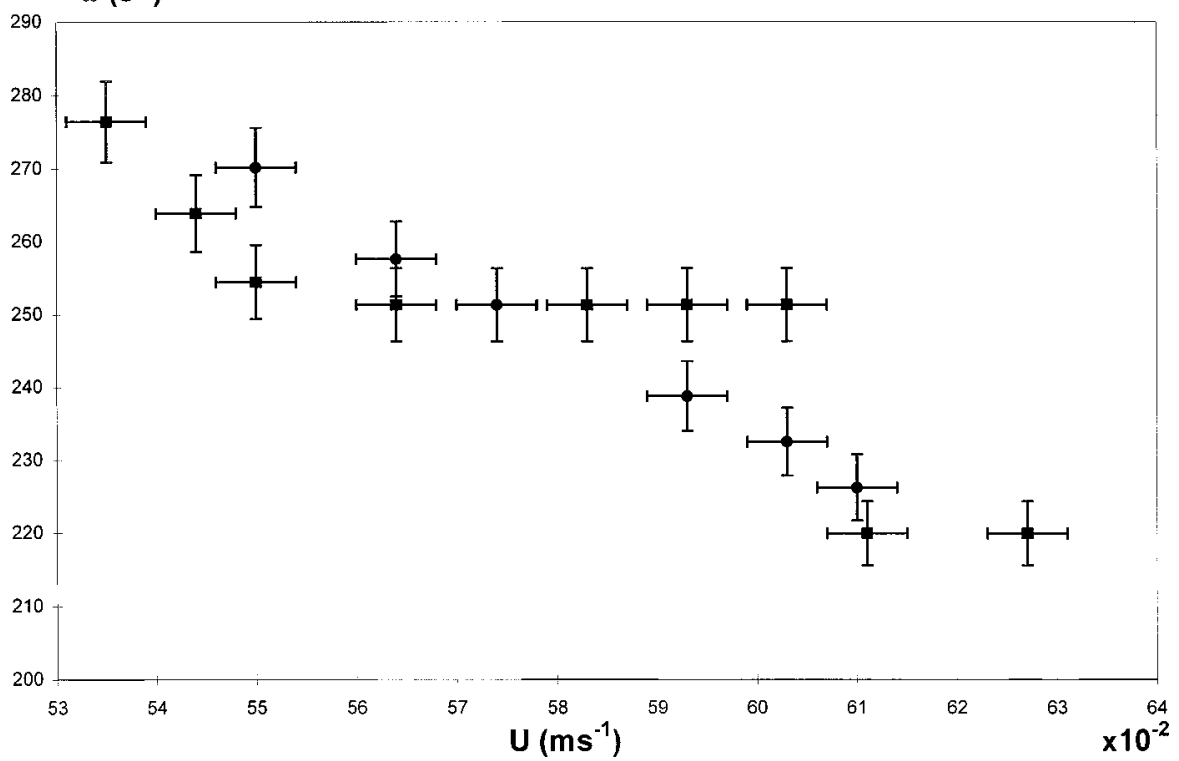

FIG. 7. Evolution of the pulsation for two coupled jets with $a=11 \mathrm{~mm}$ (squares), and for one single jet (circles) as a function of the jet velocity.

modes is accompanied by a phase and frequency jump (always positive when $\omega_{0}$ increases), which is indicated in the last column. Around the transition there is a domain where the system hesitates between the two modes with a typical half-width $\Delta \omega_{0}=10 \mathrm{~s}^{-1}$. Similar results are obtained for lines of five jets.

(ii) The second network is made of four jets displayed on squares of different sizes. The plate is still at $6 \mathrm{~mm}$ from the surface. For this network the onset of the threshold velocity is lower than for two jets so that the range of $\omega_{0}$ that can be explored is larger, between 240 and $310 \mathrm{~s}^{-1}$. As for the twojet network, bumps oscillate at the same frequency at qualitatively the same amplitude and the same direction of rotation. The two modes are recovered: in the optical mode the two adjacent jets on the square oscillate in phase opposition while in the acoustic mode all jets are in phase. Again there are transitions and a perturbed area in the transition zone. The results are presented in Table II.

\section{B. Modelization}

A natural model to fit the data should be a network of jets oscillating all at the same frequency $\omega$ with the same direction of rotation and coupled through surface waves. One considers first jet $m$. It is defined by its position $Z_{m}=X_{m}$ $+i Y_{m}$, and the bump displacement is $z_{m}=x_{m}+i y_{m}$. When the stationary state is reached, $z_{m}=\alpha_{m} e^{i \omega_{0} t}$ with $\alpha_{m}$ its

TABLE I. Modes in a two-jet array for $240<\omega_{0}<300 \mathrm{~s}^{-1}$.

\begin{tabular}{llcc}
\hline \hline$a(\mathrm{~m})$ & Phase difference & $\begin{array}{c}\omega_{0} \text { at transition } \\
\left(\mathrm{s}^{-1}\right)\end{array}$ & $\begin{array}{c}\omega \text { jump at transition } \\
\left(\mathrm{s}^{-1}\right)\end{array}$ \\
\hline 0.006 & $\pi \rightarrow 2 \pi$ & 275 & $220 \rightarrow 220+80$ \\
0.0085 & $2 \pi \rightarrow \pi$ & 280 & $226 \rightarrow 226+56$ \\
0.009 & $2 \pi \rightarrow \pi$ & 270 & $226 \rightarrow 226+56$ \\
0.011 & $\pi$ & & \\
\hline \hline
\end{tabular}

slowly varying complex amplitude and $\phi_{m}=\arg \left(\alpha_{m}\right)$ represents the phase of the motion. When jet $m$ is alone, the rotation occurs with a pulsation $\omega_{0}$ so that the argument of $\alpha_{m}$ does not depend on time. This behavior is well represented by the following equation:

$$
\frac{d \alpha_{m}}{d t}-\gamma(U)\left(1-\alpha_{m} \alpha_{m}^{*}\right) \alpha_{m}=0,
$$

where an asterisk denotes complex conjugate. The second term represents the effect of the jet instability, which amplifies the motion at a rate $\gamma, \gamma$ is real (positive when $U>U_{c}$ ), and which saturates for $\left|\alpha_{m}\right|=1$ (amplitudes have been normalized to the free jet saturation amplitude).

One considers now an array of jets, coupled through surface waves. This can be more easily studied when the bumps rotate in stationary conditions at the frequency $\omega$, i.e., when $\alpha_{m}(t)=\alpha_{0} e^{i\left(\omega-\omega_{0}\right) t}$. Note that $\omega-\omega_{0} \ll \omega_{0}$ so that $\alpha_{m}(t)$ is a slowly varying function. Surface waves have a welldefined wave number $k(\omega)$, which is the solution of the surface wave dispersion relation for deep water [in our case the product $k(\omega) \times($ depth $)$ is about 5] [16]:

$$
k(\omega) g+k(\omega)^{3} g l_{c}^{2}-\omega^{2}=0 \quad \text { with } l_{c}^{2}=\frac{\sigma}{\rho g} .
$$

A given jet $n$ radiates a spiral capillary wave, which depends on the flow velocity $U$ and on the bump displacement. Dur-

TABLE II. Modes in a four-jet array for $240<\omega_{0}<310 \mathrm{~s}^{-1}$.

\begin{tabular}{llcc}
\hline \hline$a(\mathrm{~m})$ & $\begin{array}{l}\text { Phase difference } \\
(\text { neighboring jets })\end{array}$ & $\begin{array}{c}\omega_{0} \text { at transition } \\
\left(\mathrm{s}^{-1}\right)\end{array}$ & $\begin{array}{c}\omega \text { jump at transition } \\
\left(\mathrm{s}^{-1}\right)\end{array}$ \\
\hline 0.0085 & $2 \pi \rightarrow \pi$ & 295 & $250 \rightarrow 250+40$ \\
0.009 & $\pi$ & & \\
0.011 & $\pi \rightarrow 2 \pi$ & 300 & $250 \rightarrow 250+56$ \\
\hline \hline
\end{tabular}



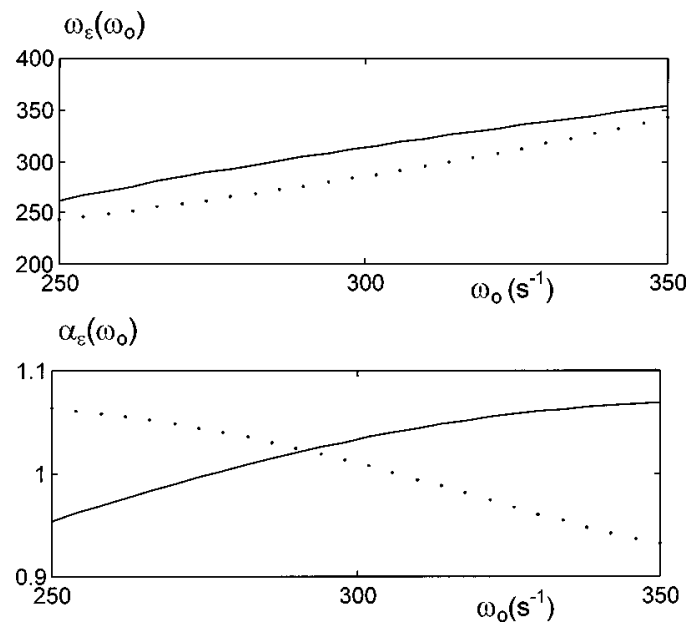

FIG. 8. Functions $\omega_{\varepsilon}\left(\omega_{0}\right)$ and $\alpha_{\varepsilon}\left(\omega_{0}\right)$ defined by Eq. (8); dotted line (acoustical mode), full line (optical mode). The numerical values chosen here are $a=8.5 \mathrm{~mm}$ and $\lambda e^{-\mu a}=15 e^{-3 i \pi / 4}$.

ing its propagation between jets $n$ and $m$ separated by a distance $r_{m n}$, the wave is attenuated by geometrical effect (energy flux proportional to $1 / r$ in cylindrical geometry) and other dissipation mechanisms. The total attenuation will be included in a function $f(r)$. The wave experiences also a phase shift given by $k(\omega) r_{m n}$. The simplest coupling term in the amplitude equation compatible with surface waves leads to the following equation:

$$
\begin{aligned}
i\left[\omega-\omega_{0}(U)\right] \alpha_{m}-\gamma(U)\left(1-\alpha_{m} \alpha_{m}^{*}\right) \alpha_{m} \\
+\lambda(U) \sum_{n \neq m} f\left(r_{m n}\right) e^{-i k(\omega) r_{m n}} \alpha_{n}=0 .
\end{aligned}
$$

The function $\omega_{0}(U)$ is the free jet frequency, which can be measured directly by single jet experiments [see Fig. 4(a)] and $\omega$ is the frequency of the motion in the array. The coupling constant $\lambda(U)$ contains the wave amplitude and the physics of the forcing effect of a wave on the helical jet instability.

This model allows us also to recover the fact that the threshold is lower when there are several jets. For instance, if one considers now in Eq. (4) a small amplitude oscillation (instead of a saturated one), the real part reads

$$
\operatorname{Im} \omega=-\gamma(U)+\operatorname{Re}\left(\lambda \sum_{n \neq m} f\left(r_{m n}\right) e^{-i k(\omega) r_{m n}}\right)=0 .
$$

Even for $U<U_{c}$ so that $\gamma$ is negative, the growth rate can be positive in accordance with the observations described in Sec. III A.

There are two parameters in our dynamical system: $\gamma(U)$ the growth rate of the free jet instability, the coupling constant $\lambda(U)$. In addition, the function $f(r)$ must be adjusted. For the sake of simplicity, it will be taken equal to $e^{-\mu r}$. The parameters $\gamma, \lambda, \mu$ do not depend on the geometry of the jet lattice (a dependence in the nozzle diameter and depth is not excluded but all experiments have been made taking these two parameters constant). In Sec. III D, experiments will be simulated fitting $\gamma(U), \lambda(U)$, and $\mu$ by simple number. Thus the parameters $\lambda$ and $\gamma$ will be taken independent of $U$, i.e., the notation "function of $U$ " will be skipped.

\section{Analytic determination of phase patterns of two- or four-jet arrays}

\section{Two-jet network}

The system is described by a set of two equations, which admit obviously solutions with $\left|z_{1}\right|=\left|z_{2}\right|=|\alpha|$. A mode is defined by the phase shift between both jets $\Delta \phi=\phi_{2}-\phi_{1}$. The system (4) becomes

$$
\begin{aligned}
& i\left(\omega-\omega_{0}\right)|\alpha|-\gamma\left(1-|\alpha|^{2}\right)|\alpha|+\lambda e^{-\mu a} e^{-i k(\omega) a}|\alpha| e^{i \Delta \phi} \\
& \quad=0, \\
& i\left(\omega-\omega_{0}\right)|\alpha|-\gamma\left(1-|\alpha|^{2}\right)|\alpha|+\lambda e^{-\mu a} e^{-i k(\omega) a}|\alpha| e^{-\Delta \phi} \\
& \quad=0 .
\end{aligned}
$$

This system has solutions if Eqs. (6a) and (6b) have the same $\omega$ as solution. Two modes are allowed, $\Delta \phi=0$ or $\Delta \phi=\pi$. Their frequencies and amplitudes are obtained from the real and imaginary parts:

$$
\begin{gathered}
\omega_{\varepsilon}-\omega_{0}+\varepsilon|\lambda| e^{-\mu a} \operatorname{Im}\left[e^{i[-k(\omega) a+\arg (\lambda)]}\right]=0, \\
-\gamma\left(1-\left|\alpha_{\varepsilon}\right|^{2}\right)+\varepsilon|\lambda| e^{-\mu a} \operatorname{Re}\left[e^{i[-k(\omega) a+\arg (\lambda)]}\right]=0
\end{gathered}
$$

with $\varepsilon$ equal to -1 or 1 , respectively, for the optical and the acoustic mode. From Eq. (7a) it is possible to deduce the frequency and amplitude of both modes. A simple approximation can be obtained using $\omega_{\varepsilon}-\omega_{0} \ll \omega_{0}$, i.e., taking the coupling terms evaluated for $\omega=\omega_{0}$ :

TABLE III. Experimental modes and simulated ones for three values of $\arg (\lambda)$.

\begin{tabular}{lllll}
\hline \hline & & \multicolumn{2}{c}{ Result of Eqs. (7) or $(9)$ for $|\lambda|=33.33$ and $\mu=120$} \\
\cline { 3 - 5 }$a(\mathrm{~m})$ jets & Transition at $\omega_{0}\left(\mathrm{~s}^{-1}\right)$ & $\arg (\lambda)=-5 \pi / 8$ & $\arg (\lambda)=-3 \pi / 4$ & $\arg (\lambda)=-7 \pi / 8$ \\
\hline $0.0062 \mathrm{~J}$ & $\pi \rightarrow 2 \pi$ at $\omega_{0}=275$ & $\pi \rightarrow 2 \pi$ at $\omega_{0}=277$ & $\pi \rightarrow 2 \pi$ at $\omega_{0}=256$ & $2 \pi$ \\
$0.00852 \mathrm{~J}$ & $2 \pi \rightarrow \pi$ at $\omega_{0}=280$ & $2 \pi \rightarrow \pi$ at $\omega_{0}=310$ & $2 \pi \rightarrow \pi$ at $\omega_{0}=291$ & $2 \pi \rightarrow \pi$ at $\omega_{0}=273$ \\
$0.0092 \mathrm{~J}$ & $2 \pi \rightarrow \pi$ at $\omega_{0}=270$ & $2 \pi \rightarrow \pi$ at $\omega_{0}=287$ & $2 \pi \rightarrow \pi$ at $\omega_{0}=270$ & $2 \pi \rightarrow \pi$ at $\omega_{0}=255$ \\
$0.0112 \mathrm{~J}$ & $\pi$ & $\pi$ & $\pi$ & $\pi \rightarrow 2 \pi$ at $\omega_{0}=300$ \\
$0.00854 J$ & $2 \pi \rightarrow \pi$ at $\omega_{0}=295$ & $2 \pi \rightarrow \pi$ at $\omega_{0}=295$ & $2 \pi \rightarrow \pi$ at $\omega_{0}=273$ & $2 \pi \rightarrow \pi$ at $\omega_{0}=255$ \\
$0.0094 J$ & $\pi$ & $2 \pi \rightarrow \pi$ at $\omega_{0}=271$ & $\pi$ & $\pi$ \\
$0.0114 J$ & $\pi \rightarrow 2 \pi$ at $\omega_{0}=300$ & $\pi \rightarrow 2 \pi$ at $\omega_{0}=320$ & $\pi \rightarrow 2 \pi$ at $\omega_{0}=311$ & $\pi \rightarrow 2 \pi$ at $\omega_{0}=300$ \\
\hline \hline
\end{tabular}


TABLE IV. Mode transitions for three values of $|\lambda|$ and $\mu$ and $\arg (\lambda)=-3 \pi / 4$.

\begin{tabular}{|c|c|c|c|c|}
\hline \multirow[b]{2}{*}{$\begin{array}{l}a(\mathrm{~m}) \\
\text { number of jets }\end{array}$} & \multirow[b]{2}{*}{$\begin{array}{l}\text { Experimental } \omega \\
\text { jump }\left(\mathrm{s}^{-1}\right)\end{array}$} & \multicolumn{3}{|c|}{ Solution of Eqs. (7) (two jets) or (9) (four jets) } \\
\hline & & $\begin{array}{c}|\lambda|=60 \\
\mu=50\end{array}$ & $\begin{array}{c}|\lambda|=100 \\
\mu=120\end{array}$ & $\begin{array}{c}|\lambda|=320 \\
\mu=250\end{array}$ \\
\hline $0.0062 J$ & $\begin{array}{l}220 \rightarrow 220+80 \\
\text { at } \omega_{0}=275 \mathrm{~s}^{-1}\end{array}$ & $\begin{array}{l}230 \rightarrow 230+70 \\
\text { at } \omega_{0}=255 \mathrm{~s}^{-1}\end{array}$ & $\begin{array}{l}220 \rightarrow 220+80 \\
\text { at } \omega_{0}=255 \mathrm{~s}^{-1}\end{array}$ & $\begin{array}{l}210 \rightarrow 210+90 \\
\text { at } \omega_{0}=255 \mathrm{~s}^{-1}\end{array}$ \\
\hline $0.00852 J$ & $\begin{array}{l}226 \rightarrow 226+56 \\
\text { at } \omega_{0}=280 \mathrm{~s}^{-1}\end{array}$ & $\begin{array}{l}250 \rightarrow 250+55 \\
\text { at } \omega_{0}=293 \mathrm{~s}^{-1}\end{array}$ & $\begin{array}{l}250 \rightarrow 250+60 \\
\text { at } \omega_{0}=290 \mathrm{~s}^{-1}\end{array}$ & $\begin{array}{l}250 \rightarrow 250+60 \\
\text { at } \omega_{0}=291 \mathrm{~s}^{-1}\end{array}$ \\
\hline $0.0092 J$ & $\begin{array}{l}226 \rightarrow 226+56 \\
\text { at } \omega_{0}=270 \mathrm{~s}^{-1}\end{array}$ & $\begin{array}{l}240 \rightarrow 240+55 \\
\text { at } \omega_{0}=270 \mathrm{~s}^{-1}\end{array}$ & $\begin{array}{l}240 \rightarrow 240+65 \\
\text { at } \omega_{0}=270 \mathrm{~s}^{-1}\end{array}$ & $\begin{array}{l}240 \rightarrow 240+60 \\
\text { at } \omega_{0}=269 \mathrm{~s}^{-1}\end{array}$ \\
\hline $0.00854 J$ & $\begin{array}{l}250 \rightarrow 250+40 \\
\text { at } \omega_{0}=295 \mathrm{~s}^{-1}\end{array}$ & $\begin{array}{l}250 \rightarrow 250+90 \\
\text { at } \omega_{0}=262 \mathrm{~s}^{-1}\end{array}$ & $\begin{array}{l}240 \rightarrow 240+80 \\
\text { at } \omega_{0}=258 \mathrm{~s}^{-1}\end{array}$ & $\begin{array}{l}230 \rightarrow 230+80 \\
\text { at } \omega_{0}=258 \mathrm{~s}^{-1}\end{array}$ \\
\hline $0.0114 J$ & $\begin{array}{l}250 \rightarrow 250+56 \\
\text { at } \omega_{0}=300 \mathrm{~s}^{-1}\end{array}$ & $\begin{array}{l}250 \rightarrow 250+90 \\
\text { at } \omega_{0}=310 \mathrm{~s}^{-1}\end{array}$ & $\begin{array}{l}260 \rightarrow 260+90 \\
\text { at } \omega_{0}=310 \mathrm{~s}^{-1}\end{array}$ & $\begin{array}{l}260 \rightarrow 260+80 \\
\text { at } \omega_{0}=312 \mathrm{~s}^{-1}\end{array}$ \\
\hline
\end{tabular}

$$
\begin{gathered}
\omega_{\varepsilon}=\omega_{0}-\varepsilon|\lambda| e^{-\mu a} \sin \left[-k\left(\omega_{0}\right) a+\arg (\lambda)\right], \\
\left|\alpha_{\varepsilon}\right|^{2}=1-\frac{\varepsilon}{\gamma}|\lambda| e^{-\mu a} \cos \left[-k\left(\omega_{0}\right) a+\arg (\lambda)\right] .
\end{gathered}
$$

For $\omega_{0}$, i.e., $U$ values so that $-k\left(\omega_{0}\right) a+\arg (\lambda)=\pi / 2(\bmod$ $\pi$ ), the two modes have the same amplitude (equal to 1 ). At these points the frequency difference between the two modes is $2|\lambda| e^{-\mu a}$. In the Appendix it is shown that only the mode that has the largest amplitude is stable. This means that experimentally only this mode should be seen. An example of this prediction is shown in Fig. 8, which illustrates one of the computations discussed in Table III. The functions $\alpha_{\varepsilon}\left(\omega_{0}\right)$ and $\omega_{\varepsilon}(0)$ are shown for $a=8.5 \mathrm{~mm}$ and $\lambda=15 e^{-3 i \pi / 4}$. According to the prescription that the observed mode is the one with the largest amplitude, a jump from acoustic to optical mode should be observed at $\omega_{0}$ around $275 \mathrm{~s}^{-1}$.

\section{Four-jet network}

One considers now a network with four jets on a square. Introducing the experimental mode structure, i.e., [0000] and $[0 \pi 0 \pi]$ in Eq. (4), one obtains the equation equivalent to Eq. (7):

$$
\begin{gathered}
\left(\omega_{\varepsilon}-\omega_{0}\right)+|\lambda| \operatorname{Im}\left(2 \varepsilon e^{-\mu a} e^{-i k(\omega) a+i \arg (\lambda)}\right. \\
\left.+e^{-\mu a \sqrt{2}} e^{-i k(\omega) a \sqrt{2}+i \arg (\lambda)}\right)=0 \\
-\gamma\left(1-\left|\alpha_{\varepsilon}\right|^{2}\right)+|\lambda| \operatorname{Re}\left(2 \varepsilon e^{-\mu a} e^{-i k(\omega) a+i \arg (\lambda)}\right. \\
\left.+e^{-\mu a \sqrt{2}} e^{-i k(\omega) a \sqrt{2}+i \arg (\lambda)}\right)=0
\end{gathered}
$$

with $\varepsilon$ equal to 1 or -1 , respectively, for the acoustic and the optical mode. These equations provide the evolution of the amplitude and the frequency of each mode with the natural frequency $\omega_{0}$. Again, within the approximation $\omega_{\varepsilon}-\omega_{0}$ $\ll \omega_{0}$, the two modes have the same amplitude for

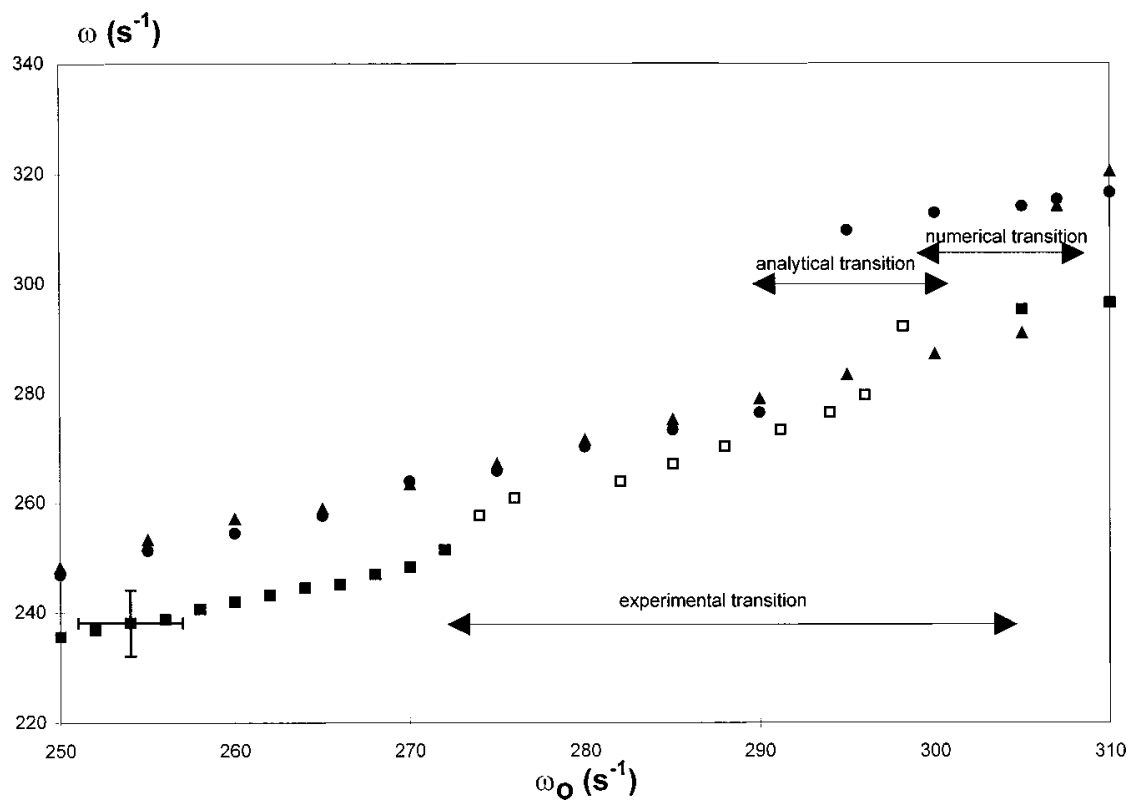

FIG. 9. Pulsation of the motion of jets displayed on a square $(a=11 \mathrm{~mm})$ as a function of the free jet pulsation $\left(\omega_{0}\right)$. Experimental results for a depth $=6 \mathrm{~mm}$ (squares), solution of Eq. (8) (circles), numerical simulation (triangles), with $\mu=120, \gamma=60, \lambda=33.3 e^{-i \times 3 \pi / 4}$. The lines with double arrows on the graph represent the transition areas between the acoustic and the optical mode. 
$-k\left(\omega_{0}\right) a+\arg (\lambda)=\pi / 2(\bmod \pi)$, while the frequency difference at the transition between the two modes is $4|\lambda| e^{-\mu a}$.

\section{COMPARISON WITH THE EXPERIMENTAL DATA}

\section{A. Mode transition}

In Table III, one shows a comparison between the experimental data and the ones obtained with the analytical treatment for values of $\arg (\lambda)$ in an optimum range, i.e., around $-3 \pi / 4$. The second column of Table III contains experimental data from Sec. III A while the last three columns give the modes with the largest amplitudes obtained from Eqs. (7) or (9) $\arg (\lambda)$ has been determined by trying to fit all the observed transitions (their location depends on $|\lambda|$ only through corrections of order $\left(\omega-\omega_{0}\right) / \omega_{0}$, as can be seen in Table IV), or at least the observed modes when the values of jet spacing $a$ correspond to windows without transition. The computations are made in the window $250<\omega_{0}<325 \mathrm{~s}^{-1}$ for two and four jets. The predicted modes are in quite good agreement with the experimental ones, especially if one takes into account the error bars about $+/-10 \mathrm{~s}^{-1}$ on $\omega_{0}$ at transition. In the following $\arg (\lambda)=-3 \pi / 4$ will be taken.

\section{B. Strength of the coupling}

In a second step, $|\lambda|$ and $\mu$ can be found from expression (7a) using the two-jet experiment. For both modes, experimental frequencies should follow the relation

$$
\ln \left|\frac{\omega^{2}-\omega_{0}^{2}}{\cos (k(\omega) a+\pi / 4)}\right|=\ln |\lambda|-\mu a .
$$

If the value of $\omega$ can be measured as a function of $a$, it should be possible to guess $|\lambda|$ and $\mu$. However, due to the small range of $a$ and the low value of attenuation, the accuracy is poor. Several combinations of $\lambda$ and $\mu$ are possible provided $|\lambda| e^{-a \mu}$ stays in the same range. This is shown in Table IV, which displays a comparison between the five experimental mode transitions given in Tables I and II and the simulated ones taking two combinations of $|\lambda|$ and $\mu$.

To summarize, from Tables III and IV, it appears that the amplitude, nature, and location of modes can be reasonably fitted by the model especially if one takes into account the small number of parameters to be fitted: One $[\arg (\lambda)]$ to fit all the transition (location and direction) and two [modu$\operatorname{lus}(\lambda)$ and $\mu]$ for the values of frequencies (with little effect). The value of $\gamma$ plays almost no role (it has been taken equal to 60). An example of comparison theory-experiment of $\omega\left(\omega_{0}\right)$ for a four-jet system is shown in Fig. 9. The transition has been simulated in two ways. First there is the largest amplitude mode given by Eq. (9). One admits then that the system cannot choose between the two modes if it exists a difference of less than $10 \%$ between the amplitudes of the modes. This assumption allows us to determine a small transition zone ("analytical transition") where the two modes, like in the experiments, are unstable. This is possibly due to desynchronization as discussed in the next section. Second, there is the result obtained by evolving in time the jet motion using a scheme described elsewhere [17].

\section{Effect of a scattering of oscillator properties}

When $a$ increases beyond $12 \mathrm{~mm}$, the synchronization becomes poor and eventually is lost. Another experimental feature is that, in the transition zone (when the amplitudes of the two modes are close together) the system is unstable. A possible explanation could be the unavoidable dispersion in the $\omega_{0}$ values, which tends to hamper the synchronization. This is a well known effect for coupled oscillators [1]. This effect has been investigated more quantitatively for a two-jet system. One assumes that there is some scatter in the $\omega_{0}$ values, i.e., that they become $\omega_{1,2}=\omega_{0} \pm \Delta$. In the presence of coupling, the motion of jet $m$ can be expressed as $\alpha_{m}(t) e^{-i \omega_{0} t}$. Provided it is slowly varying, the evolution of $\alpha_{m}(t)$ obeys the following equation:

$$
\begin{gathered}
\frac{d \alpha_{m}}{d t}-i\left(\omega_{m}-\omega_{0}\right) \alpha_{m}-\gamma\left(1-\alpha_{m} \alpha_{m}^{*}\right) \alpha_{m} \\
+\lambda \sum_{n \neq m} e^{-\mu a} e^{-i k\left(\omega_{n}\right) r_{m n}} \alpha_{n}=0 .
\end{gathered}
$$

Equation (11) has been solved using an iterative scheme to determine the $\omega_{n}$ values to be used in the coupling coefficients [17]. For the system chosen ( $a=8.5 \mathrm{~mm}$ as in Fig. 8) there is a predicted transition acoustic $\rightarrow$ optical at $\omega_{0}$ $=291 \mathrm{~s}^{-1}$ (observed at $\left.280 \mathrm{~s}^{-1}\right)$. For $\Delta=0$, these modes are found by the evolutive computation. When $\Delta$ is nonzero, depending on the $\lambda e^{-\mu a}$ value, there exists a zone $\Delta$ $<\Delta_{\max }$ where a synchronization is possible. The value of $\Delta_{\text {max }}$ depends on $k\left(\omega_{0}\right) a+\arg (\lambda)$ and $\left|\lambda e^{-\mu a}\right|$. This domain is displayed in Fig. 10(a) for $\omega_{0}=240 \mathrm{~s}^{-1}$ (far from the mode transition) and $295 \mathrm{~s}^{-1}$ (close to the mode transition). The synchronization domain depends on $k\left(\omega_{0}\right) a$, i.e., the phase of the interaction between the jets. In particular, close to the transition point, the system becomes very sensitive to any defect, i.e., is hard to synchronize. This is expected since at this location the eigenvalues associated to the mode stability analysis are close to zero (see the Appendix). This is shown in Fig. 10(b), which displays the value of $\Delta_{\max }$ for $\left|\lambda e^{-\mu}\right|=15$ as a function of $\omega_{0}$. This simple model explains two experimental findings. (i) The synchronization becomes difficult for $a>12 \mathrm{~mm}$. According to Table IV, the values of $\lambda e^{-\mu a}$ at this point range between $16(\mu=120)$ and $32(\mu$ $=50$ ). For such coupling parameters, according to Fig. 10 (b), the desynchronization occurs for $\Delta$ about $10-20 \mathrm{~s}^{-1}$, i.e., an $\omega_{0}$ scatter in the range $5-10 \%$, i.e., according to Eq. (1) a scatter in the hole diameter of order of $5 \%$. (ii) The width of the unstable domain in Fig. 10(b) is compatible with the width of the transition zone in experiments.

\section{CONCLUSION}

The mechanism for the oscillation of a single vertical jet impinging on an interface has been investigated. It turns out that a feedback mechanism between surface waves and the unstable jet is at the origin of the oscillations of the bump to the surface. An analogy can be made with the "jet-edge" system if considering a "resonant edge" mechanically attached to springs. Indeed, the stiffness of the springs is here equivalent to the surface tension of the fluid. In a dimensional model, the frequency is found to be proportional to the 
$Y=\Delta$

(a)

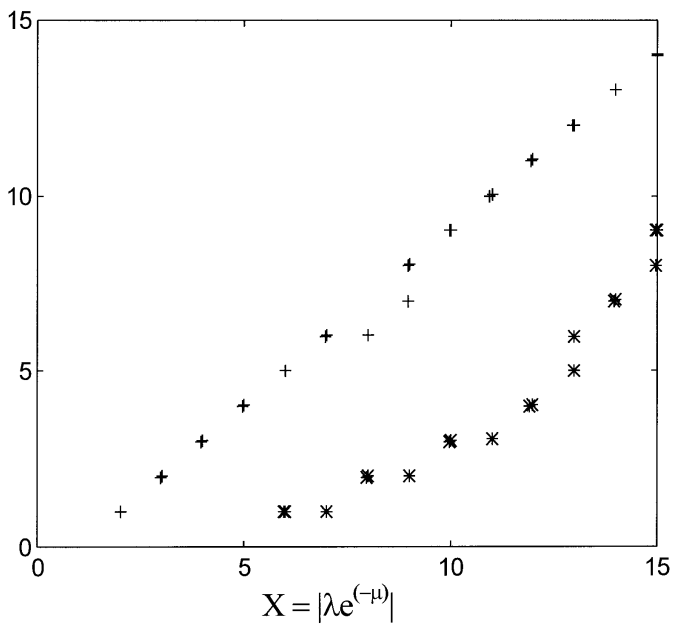

$\mathrm{Y}=\Delta_{\max }$

(b)

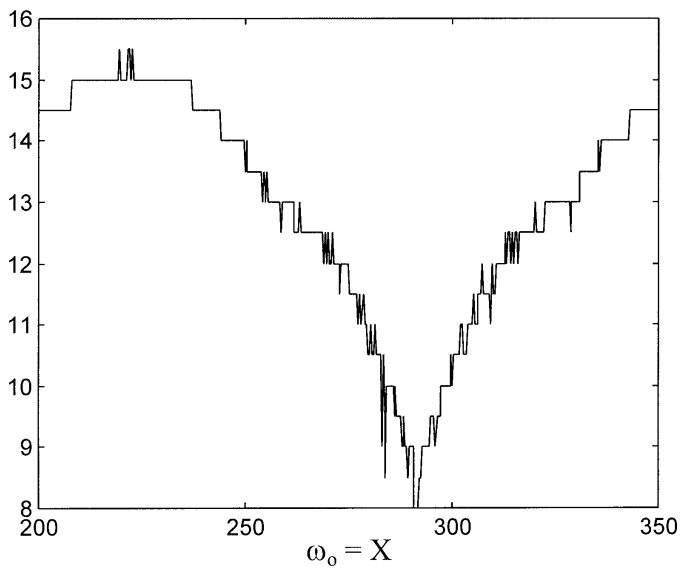

FIG. 10. (a) Synchronization domain for $\omega_{0}=240 \mathrm{~s}^{-1}$ (crosses) and $295 \mathrm{~s}^{-1}$ (stars) for a two-jet system with $a=8.5 \mathrm{~mm}$ in the space $X=\left|\lambda e^{-\mu}\right|$ and $Y=\Delta ;[\arg (\lambda)$ is kept equal to $-3 i \pi / 4]$. (b) Value of $Y=\Delta_{\max }$ as a function of $X=\omega_{0}$ for $\lambda e^{-\mu}=15 e^{-3 i \pi / 4}$ and $a=8.5 \mathrm{~mm}$.

surface tension and conversely proportional to the jet velocity. These results are in good agreement with the experimental results obtained when varying the surface tension of the fluid, the velocity of the jet, and the depth of the nozzle.

Considering simple ensembles of jets, a phase-locking mechanism has been observed. When the jet velocity, i.e., the resonant frequency is modified and two modes are observed, namely an acoustic mode and an optical one. A modelization in which each bump is a nonlinear oscillator coupled with its neighbors through surface waves gives reasonable agreement with the experimental results, especially if one takes into account the scatter of jet frequencies due to imperfections. Generalization of this model to infinite networks is possible at the expense of a more complex formalism, which will be presented in a forthcoming paper [17]. It appears that systems of jets are very close to the large population of coupled oscillators investigated by theoreticians. There is, however, one important difference, the coupling is ensured not by instantaneous interaction (reflected by a coupling constant) but by traveling waves propagating at finite velocity. This plays a significant role in the formation of phase patterns observed in large regular networks.

\section{APPENDIX: STABILITY ANALYSIS OF THE LIMIT CYCLE IN THE TWO JET CONFIGURATION}

Following the notation of Sec. III C, the two jet system has two limit cycles described by Eq. (6), the acoustic mode with an amplitude at saturation $\alpha_{1}$ and a frequency $\omega_{1}$ and the optical mode with an amplitude $\alpha_{-1}$ and a frequency $\omega_{-1}$. The phase shift between the bumps of a given mode is $\phi_{\varepsilon}$, which is equal to zero for $\varepsilon=1$ and $\pi$ otherwise. The equations governing the eigenmodes are

$$
i\left(\omega_{\varepsilon}-\omega_{0}\right)-\gamma\left(1-\alpha_{\varepsilon} \alpha_{\varepsilon}^{*}\right)+\lambda e^{-\mu a-i k\left(\omega_{\varepsilon}\right) a} e^{i \phi_{\varepsilon}}=0 .
$$

One considers that the system is at the limit cycle for the mode $\varepsilon(=+$ or -1$)$ so that the envelope of rotation of bump $m(m=1,2)$ is close to $\alpha_{\varepsilon} e^{i\left(\omega_{\varepsilon}-\omega_{0}\right) t+i m \phi_{\varepsilon}}$. A small perturbation corresponding to a mode $\varepsilon^{\prime} \neq \varepsilon$ is added which is of the form $f(t) e^{i\left(\omega_{\varepsilon}^{\prime}-\omega_{0}\right) t+i m \phi_{\varepsilon^{\prime}}}$. Thus, the envelope of the position of bump $m$ is $\alpha_{m}=\alpha_{\varepsilon} e^{\left[i\left(\omega_{\varepsilon}-\omega_{0}\right) t+i m \phi_{\varepsilon}\right]}$ $+f(t) e^{i\left(\omega_{\varepsilon}^{\prime}-\omega_{0}\right) t+i m \phi_{\varepsilon}}$ and is as follows:

$$
\begin{gathered}
\frac{d \alpha_{m}}{d t}-\gamma\left(1-\alpha_{m} \alpha_{m}^{*}\right) \alpha_{m}+\lambda e^{-\mu a-i \omega_{0} t}\left[e^{-i k\left(\omega_{\varepsilon}\right) a} \alpha_{\varepsilon} e^{i\left(\omega_{\varepsilon} t+\phi_{\varepsilon}\right)}\right. \\
\left.+e^{-i k\left(\omega_{\varepsilon^{\prime}}\right) a} f(t) e^{i\left(\omega_{\varepsilon}^{\prime} t+\phi_{\varepsilon^{\prime}}\right)}\right]=0 .
\end{gathered}
$$

The zero-order equation gives the equation of the limit cycle of mode $\varepsilon$. The terms can then be developed to first order in $f$. The linearized terms are

$$
\begin{gathered}
\frac{d \alpha_{m}}{d t} \rightarrow\left(\frac{d f}{d t}+i\left(\omega_{\varepsilon}^{\prime}-\omega_{0}\right) f(t)\right) e^{i\left(\omega_{\varepsilon}^{\prime}-\omega_{0}\right) t+i m \phi_{\varepsilon^{\prime}},} \\
\alpha_{m} \alpha_{m}^{*} \alpha_{m} \rightarrow \alpha_{\varepsilon}^{2} e^{i\left(\omega_{\varepsilon}^{\prime}-\omega_{0}\right) t+i m \phi_{\varepsilon^{\prime}}}\left[2 f(t)+f^{*}(t) e^{2 i \theta(t)}\right]
\end{gathered}
$$

with $\theta(t)=\left(\omega_{\varepsilon}-\omega_{\varepsilon}^{\prime}\right) t+m\left(\phi_{\varepsilon}-\phi_{\varepsilon^{\prime}}\right)$.

After multiplication by $e^{-i \omega_{\varepsilon}^{\prime} t-i m \phi_{\varepsilon}^{\prime}}$, an evolution equation for $f(t)$ can be deduced from the linearized equation (A1),

$$
\begin{aligned}
\frac{d f}{d t}+ & i\left(\omega_{\varepsilon}^{\prime}-\omega_{0}\right) f(t)-\gamma f(t)+2 \gamma \alpha_{\varepsilon}^{2} f(t) \\
& +\lambda f(t) e^{-\mu a} e^{-i k\left(\omega_{\varepsilon}^{\prime}\right) a+i \phi_{\varepsilon^{\prime}}}=-\gamma \alpha_{\varepsilon}^{2} f^{*}(t) e^{2 i \theta(t)} .
\end{aligned}
$$

Using Eq. (A1) expressed for the limit cycle of mode $\varepsilon^{\prime}$, Eq. (A3) can be simplified. Taking the complex conjugate, a similar equation can be obtained for $f^{*}(t)$,

$$
\frac{d f}{\gamma d t}=\left(\alpha_{\varepsilon^{\prime}}^{2}-2 \alpha_{\varepsilon}^{2}\right) f(t)-\alpha_{\varepsilon}^{2} f^{*}(t) e^{2 i \theta(t)},
$$




$$
\frac{d f^{*}}{\gamma d t}=-\alpha_{\varepsilon}^{2} f(t) e^{-2 i \theta(t)}+\left(\alpha_{\varepsilon^{\prime}}^{2}-2 \alpha_{\varepsilon}^{2}\right) f^{*}(t)
$$

Since $\theta(t)$ is a slowly varying function, the system can be considered as a linear operator on $f$. The eigenvalues $\lambda$ are given by the zeros of the determinant $\lambda^{2}-2 \lambda\left(\alpha_{\varepsilon^{\prime}}^{2}-2 \alpha_{\varepsilon}^{2}\right)$ $+\left(2 \alpha_{\varepsilon}^{2}-\alpha_{\varepsilon^{\prime}}^{2}\right)^{2}-\alpha_{\varepsilon}^{4}$. The roots are $\alpha_{\varepsilon^{\prime}}^{2}-3 \alpha_{\varepsilon}^{2}$ and $\alpha_{\varepsilon^{\prime}}^{2}-\alpha_{\varepsilon}^{2}$. Both roots have a negative real part, i.e., the system is stable if and only if the limit cycle corresponds to the mode with the largest amplitude.
[1] P. C. Matthews, R. E. Mirollo, and S. H. Strogatz, Physica D 52, 293 (1991).

[2] N. Nakagawa and Y. Kuramoto, Prog. Theor. Phys. 89, 313 (1993).

[3] V. Hakim and W.-J. Rappel, Phys. Rev. A 46, 7347 (1992).

[4] P. Legal, M. P. Chauve, I. Peshard, and S. Jarre, Curr. Top. Phys. Fluids Res. Trends 1, 307 (1994); I. Peshard and P. Le Gal, Phys. Rev. Lett. 77, 3122 (1996).

[5] E. Villermaux and E. J. Hopfinger, Physica D 72, 230 (1994); J. Fluid Mech. 263, 63 (1994).

[6] H. Willaime, O. Cardoso, and P. Tabeling, Phys. Rev. Lett. 67, 3247 (1991).

[7] E. Villermaux, Phys. Rev. Lett. 75, 4618 (1995).

[8] A. Maurel, S. Cremer, and P. Jenffer, Europhys. Lett. 39, 503 (1997).
[9] S. Houard, F. Daviaud, and P. Bergé, Europhys. Lett. 32, 101 (1995).

[10] S. Houard, F. Daviaud, and P. Bergé, Physica D 99, 318 (1997).

[11] G. E. Mattingly and C. C. Chang, J. Fluid Mech. 65, 541 (1974).

[12] P. J. Morris, J. Fluid Mech. 77, 511 (1976).

[13] A. Powell, J. Acoust. Soc. Am. 33, 395 (1961); D. Rockwell and E. Naudascher, Annu. Rev. Fluid Mech. 11, 67 (1979); D. G. Crighton, J. Fluid Mech. 234, 361 (1992).

[14] S. M. Kusek, T. C. Corke, and P. Reisentel, Exp. Fluids 10, 116 (1990).

[15] T. Staubli and D. Rockwell, J. Fluid Mech. 176, 135 (1987).

[16] L. D. Landau and E. M. Lifshitz, Fluid Mechanics (Pergamon, Oxford, 1987).

[17] F. Giorgiutti and L. Laurent (unpublished). 